\title{
AN OPTIMAL SEQUENTIAL PROCEDURE FOR A BUYING-SELLING PROBLEM WITH INDEPENDENT OBSERVATIONS
}

\author{
G. SOFRONOV, ${ }^{* * *}$ \\ JONATHAN M. KEITH ${ }^{* * * *}$ AND \\ DIRK P. KROESE, ${ }^{* * * * *}$ The University of Queensland
}

\begin{abstract}
We consider a buying-selling problem when two stops of a sequence of independent random variables are required. An optimal stopping rule and the value of a game are obtained.

Keywords: Optimal stopping; multiple stopping rule; value of a game; buying-selling problem

2000 Mathematics Subject Classification: Primary 60G40; 62L15

Secondary 62P20; 91B26
\end{abstract}

\section{Statement of the problem}

Let $y_{1}, y_{2}, \ldots, y_{N}$ be a sequence of random variables, where $y_{n}=f_{n}+\varepsilon_{n}, f_{n}=f(n), n=$ $1,2, \ldots, N$, is a known deterministic function (for example, a trend), and $\left\{\varepsilon_{n}, n=1, \ldots, N\right\}$ is a sequence of independent random variables with $\mathrm{E} \varepsilon_{n}=0$. The random variable $y_{n}$ can be interpreted as the value of an asset at time $n$. We observe these random variables sequentially and have to decide when we must stop. The first stop means the buying of an asset and the second stop signifies the selling of an asset. Our decision to stop depends on the observations already made, but does not depend on the future, which is not yet known. After two stops at times $m_{1}$ and $m_{2}, 1 \leq m_{1}<m_{2} \leq N$, we get a gain $Z_{m_{1}, m_{2}}=y_{m_{2}}-y_{m_{1}}$. If we do not buy anything until time $N$ then we get the gain $Z_{m_{1}, m_{2}}=0$, so we may assume that $m_{1}=N$ and $m_{2}=N+1$. This implies that

$$
\begin{gathered}
1 \leq m_{1} \leq N_{1}, \quad N_{1}=N, \\
m_{1}<m_{2} \leq N_{2}\left(m_{1}\right), \quad N_{2}\left(m_{1}\right)= \begin{cases}N & \text { if } m_{1}<N, \\
N+1 & \text { if } m_{1}=N .\end{cases}
\end{gathered}
$$

The problem consists of finding a procedure for maximizing the expected gain. Notice that problems with one stop (either buying or selling) were comprehensively considered in [4].

\section{Some results of the theory of optimal multiple stopping rules}

Let $y_{1}, y_{2}, \ldots$ be a sequence of random variables with known joint distribution. We are allowed to observe the $y_{n}$ sequentially, stopping anywhere we please. If we stop at time $m_{1}$ after

Received 21 December 2005; revision received 7 February 2006.

* Postal address: Department of Mathematics, The University of Queensland, Brisbane, QLD 4072, Australia.

** Email address: georges@ maths.uq.edu.au

*** Email address: j.keith1@uq.edu.au

**** Email address: kroese@maths.uq.edu.au 
observations $\left(y_{1}, \ldots, y_{m_{1}}\right)$, then we begin to observe another sequence $y_{m_{1}, m_{1}+1}, y_{m_{1}, m_{1}+2}, \ldots$ (depending on $\left(y_{1}, \ldots, y_{m_{1}}\right)$ ), and we must solve the problem of an optimal stopping of the new sequence. If we make $i$ stops at times $m_{1}, m_{2}, \ldots, m_{i}, 1 \leq i \leq k-1$, then we observe a sequence of random variables $y_{m_{1}, \ldots, m_{i}, m_{i}+1}, y_{m_{1}, \ldots, m_{i}, m_{i}+2}, \ldots$ whose distribution depends on $\left(y_{1}, \ldots, y_{m_{1}}, y_{m_{1}, m_{1}+1}, \ldots, y_{m_{1}, m_{2}}, \ldots, y_{m_{1}, \ldots, m_{i}}\right)$. Our decision to stop at times $m_{i}, i=$ $1,2, \ldots, k$, depends solely on the values of the basic random sequence already observed and not on any future values. After $k, k \geq 2$, stops, we receive the gain

$$
Z_{m_{1}, \ldots, m_{k}}=g_{m_{1}, \ldots, m_{k}}\left(y_{1}, \ldots, y_{m_{1}, m_{1}+1}, \ldots, y_{m_{1}, \ldots, m_{k}}\right),
$$

where $g_{m_{1}, \ldots, m_{k}}$ is a known function. We are interested in finding stopping rules which maximize our expected gain.

More formally, assume that we are given

(a) a probability space $(\Omega, \mathcal{F}, P)$;

(b) a nondecreasing sequence of $\sigma$-subalgebras $\left\{\mathcal{F}_{m_{1}, \ldots, m_{i-1}, m_{i}}, m_{i}>m_{i-1}\right\}$ of $\sigma$-algebra $\mathcal{F}$ such that

$$
\mathcal{F}_{m_{1}, \ldots, m_{i-1}} \subseteq \mathcal{F}_{m_{1}, \ldots, m_{i}} \subseteq \mathcal{F}_{m_{1}, \ldots, m_{i-1}, m_{i}+1},
$$

for all $i=1,2, \ldots, k$, with $0=: m_{0}<m_{1}<\cdots<m_{i-1}$;

(c) a random process

$$
\left\{Z_{m_{1}, \ldots, m_{k-1}, m_{k}}, \mathcal{F}_{m_{1}, \ldots, m_{k-1}, m_{k}}, m_{k}>m_{k-1}\right\},
$$

for any fixed integer $m_{1}, \ldots, m_{k-1}, 1 \leq m_{1}<m_{2}<\cdots<m_{k-1}$.

In terms of the informal background of the first paragraph in this section, we can express the $\sigma$-algebra as follows:

$$
\mathcal{F}_{m_{1}, \ldots, m_{i}}=\sigma\left(y_{1}, \ldots, y_{m_{1}}, y_{m_{1}, m_{1}+1}, \ldots, y_{m_{1}, m_{2}}, \ldots, y_{m_{1}, m_{2}, \ldots, m_{i}}\right) .
$$

Following [3], we now give the required definitions and theorems.

Definition 1. A collection of integer-valued random variables $\left(\tau_{1}, \ldots, \tau_{i}\right)$ is called an $i$-multiple stopping rule, $1 \leq i \leq k$, if the following conditions hold:

(a) $1 \leq \tau_{1}<\tau_{2} \cdots<\tau_{i}<\infty$ (P-a.s. (almost surely)),

(b) $\left\{\omega: \tau_{1}=m_{1}, \ldots, \tau_{j}=m_{j}\right\} \in \mathcal{F}_{m_{1}, \ldots, m_{j}}$, for all $m_{j}>m_{j-1}>\cdots>m_{1} \geq 1$, $j=1,2, \ldots, i$.

A $k$-multiple stopping rule with $k>1$ is called a multiple stopping rule.

We use the following notation, where $\xi$ represents an arbitrary random variable:

$$
\begin{aligned}
(\boldsymbol{m})_{i} & =\left(m_{1}, m_{2}, \ldots, m_{i}\right), \quad(\boldsymbol{m})_{1}=m_{1}, \\
\mathrm{E}_{(\boldsymbol{m})_{i}} \xi & =\mathrm{E}\left(\xi \mid \mathcal{F}_{(\boldsymbol{m})_{i}}\right), \\
A_{(\boldsymbol{m})_{i}} \xi & =\mathrm{E}_{(\boldsymbol{m})_{i}}\left(\sup _{m_{i+1}} \mathrm{E}_{(\boldsymbol{m})_{i+1}}\left(\cdots\left(\sup _{m_{k-1}} \mathrm{E}_{(\boldsymbol{m})_{k-1}} \xi\right) \cdots\right)\right) .
\end{aligned}
$$

The following condition is needed for the existence of all considered expectations. 


\section{Condition 1.}

$$
\mathrm{E}\left(\sup _{m_{1}} A_{(\boldsymbol{m})_{1}}\left(\sup _{(\boldsymbol{m})_{k}} Z_{(\boldsymbol{m})_{k}}\right)\right)<+\infty .
$$

We assume that Condition 1 is satisfied for the $Z_{(\boldsymbol{m})_{k}}$.

Let $S_{m}$ be a class of multiple stopping rules $\tau=\left(\tau_{1}, \ldots, \tau_{k}\right)$ such that $\tau_{1} \geq m$ (P-a.s.).

Definition 2. The function

$$
v_{m}=\sup _{\boldsymbol{\tau} \in S_{m}} \mathrm{E} Z_{\boldsymbol{\tau}}
$$

is called the $m$-value of the game. In particular, if $m=1$ then $v=v_{1}$ is called the value of the game.

Definition 3. A multiple stopping rule $\tau^{*} \in S_{m}$ is called an optimal multiple stopping rule in $S_{m}$ if $\mathrm{E} Z_{\boldsymbol{\tau}^{*}}$ exists and $\mathrm{E} Z_{\boldsymbol{\tau}^{*}}=v_{m}$.

Condition 1 ensures the finiteness of $v_{m}$ and the existence of $\mathrm{E} Z_{\boldsymbol{\tau}}$ for all $\boldsymbol{\tau} \in S_{m}$. The problem consists of finding an optimal multiple stopping rule and an $m$-value of the game $v_{m}$.

The sequences $\left\{V_{(\boldsymbol{m})_{i}}\right\}$ and $\left\{X_{(\boldsymbol{m})_{i}}\right\}, i=1,2, \ldots, k$, are needed for constructing the multiple stopping rules $\boldsymbol{\tau}^{*}$. Let $T_{(\boldsymbol{m})_{i}}$ be a class of $i$-multiple stopping rules $(\boldsymbol{\tau})_{i}=\left(\tau_{1}, \ldots, \tau_{i}\right)$, $i=1,2, \ldots, k$, with $\tau_{1}=m_{1}, \ldots, \tau_{i-1}=m_{i-1}, \tau_{i} \geq m_{i}$ (P-a.s.). Let $T_{(\boldsymbol{m})_{1}}=: T_{m_{1}}$ denote the class of all stopping times $\tau_{1}$ such that $\tau_{1} \geq m_{1}$ (P-a.s.). We set $X_{(\boldsymbol{m})_{k}}=Z_{(\boldsymbol{m})_{k}}$ and define, by backward induction on $i$ from $i=k$,

$$
\begin{aligned}
V_{(\boldsymbol{m})_{i}} & =\underset{(\boldsymbol{\tau})_{i} \in T_{(\boldsymbol{m})_{i}}}{\operatorname{ess} \sup _{(\boldsymbol{m})_{i}}} X_{(\boldsymbol{\tau})_{i}}, \\
X_{(\boldsymbol{m})_{i-1}} & =\mathrm{E}_{(\boldsymbol{m})_{i-1}} V_{(\boldsymbol{m})_{i-1}, m_{i-1}+1}, \quad i=k, k-1, \ldots, 1,
\end{aligned}
$$

where $X_{0}:=0$.

Remark 1. We emphasize that most of the statements in this section are valid almost surely. We shall make no mention of this in what follows.

Let us now establish some properties of the sequences $\left\{V_{(\boldsymbol{m})_{i}}\right\}$ and $\left\{X_{(\boldsymbol{m})_{i}}\right\}$. It follows from results of the general theory of optimal stopping (see, for example, [1] and [2]) that $V_{(\boldsymbol{m})_{i}}$ satisfies the recursion equation

$$
V_{(\boldsymbol{m})_{i}}=\max \left\{X_{(\boldsymbol{m})_{i}}, \mathrm{E}_{(\boldsymbol{m})_{i}} V_{(\boldsymbol{m})_{i-1}, m_{i}+1}\right\}
$$

The following theorem gives the existence conditions and the structure of an optimal multiple stopping rule in $S_{m}$.

Theorem 1. ([3].) Let Condition 1 be satisfied. We put

$$
\tau_{i}^{*}=\inf \left\{m_{i}>m_{i-1}: V_{(\boldsymbol{m})_{i}}=X_{(\boldsymbol{m})_{i}}\right\}
$$


for $i=1,2, \ldots, k$, on the set $D_{i-1}=\left\{\omega: \tau_{1}^{*}=m_{1}, \ldots, \tau_{i-1}^{*}=m_{i-1}\right\}$, where it is assumed that $\tau_{i}^{*}(\omega)=+\infty$ on $\left\{\omega: \tau_{i-1}^{*}(\omega)=+\infty\right\}, m_{0}=m-1$, and $D_{0}=\Omega$. In that case, if the random vector $\tau^{*}=\left(\tau_{1}^{*}, \ldots, \tau_{k}^{*}\right)$ is finite with probability one, then $\tau^{*} \in S_{m}$ is an optimal multiple stopping rule.

The following theorem gives the characterization of the $m$-value $v_{m}$ by means of the sequence $\left\{V_{m}\right\}$.

Theorem 2. ([3].) If Condition 1 is satisfied, then $v_{m}=\mathrm{E} V_{m}$.

We now consider a finite case. Let

$$
\left\{Z_{(\boldsymbol{m})_{k}}, 1 \leq m_{1} \leq N_{1}, m_{1}<m_{2}<N_{2}\left(m_{1}\right), \ldots, m_{k-1}<m_{k} \leq N_{k}\left(m_{1}, \ldots, m_{k-1}\right)\right\}
$$

be a family of random variables, where $N_{1}$ and $N_{i}(\cdot), i=2, \ldots, k$, are natural numbers. As in the general theory of optimal stopping [1], we define the sequence $V_{(\boldsymbol{m})}$ by backward induction from the recursion equations

$$
\begin{aligned}
V_{(\boldsymbol{m})_{i-1}, N_{i}\left(m_{1}, \ldots, m_{i-1}\right)} & =X_{(\boldsymbol{m})_{i-1}, N_{i}\left(m_{1}, \ldots, m_{i-1}\right)}, \\
V_{(\boldsymbol{m})_{i}} & =\max \left\{X_{(\boldsymbol{m})_{i}}, \mathrm{E}_{(\boldsymbol{m})_{i}} V_{(\boldsymbol{m})_{i-1}, m_{i}+1}\right\},
\end{aligned}
$$

for $1 \leq m_{1} \leq N_{1}, \ldots, m_{i-1}<m_{i} \leq N_{i}\left(m_{1}, \ldots, m_{i-1}\right)$. As before, $X_{(\boldsymbol{m})_{k}}=Z_{(\boldsymbol{m})_{k}}$.

Using Theorem 1, we define the optimal multiple stopping rule $\boldsymbol{\tau}^{*}$. From Theorem 2, (2), and (3), we obtain the value $v_{m}$.

\section{Main result}

Let us consider our initial problem. The following theorem gives an optimal double stopping rule $\tau^{*}$ and the value $v$.

Theorem 3. Let $\left\{y_{n}\right\}$ and $Z_{m_{1}, m_{2}}$ be defined as in Section 1. Let $v^{L, l}$ be the value of a game with $l, l \leq 2$, stops and $L, L \leq N$, steps. Then the value $v$ is equal to $v^{N, 2}$, where

$$
\begin{aligned}
& v^{n, 2}=\mathrm{E}\left(\max \left\{v^{n-1,1}-y_{N-n+1}, v^{n-1,2}\right\}\right), \quad 2 \leq n \leq N, v^{1,2}=0, \\
& v^{n, 1}=\mathrm{E}\left(\max \left\{y_{N-n+1}, v^{n-1,1}\right\}\right), \quad 1 \leq n \leq N-1, v^{0,1}=-\infty .
\end{aligned}
$$

We put

$$
\begin{aligned}
& \tau_{1}^{*}=\min \left\{\min \left\{m_{1}: 1 \leq m_{1} \leq N-1, y_{m_{1}} \leq v^{N-m_{1}, 1}-v^{N-m_{1}, 2}\right\}, N\right\}, \\
& \tau_{2}^{*}=\min \left\{\min \left\{m_{2}: \tau_{1}^{*}<m_{2} \leq N, y_{m_{2}} \geq v^{N-m_{2}, 1}\right\},\left\{N+1: \tau_{1}^{*}=N\right\}\right\} ;
\end{aligned}
$$

then $\tau^{*}=\left(\tau_{1}^{*}, \tau_{2}^{*}\right)$ is the optimal double stopping rule.

Proof. If $m_{1}=N$ and $m_{2}=N+1$, then $Z_{m_{1}, m_{2}}=0$. It follows easily that $v^{1,2}=0$.

Let us consider the case $1 \leq m_{1} \leq N-1, m_{1}<m_{2} \leq N$. From (3) and the independence of $y_{1}, \ldots, y_{N}$, we obtain

$$
\begin{aligned}
V_{m_{1}, m_{2}} & =\max \left\{X_{m_{1}, m_{2}}, \mathrm{E}_{m_{1}, m_{2}} V_{m_{1}, m_{2}+1}\right\} \\
& =\max \left\{y_{m_{2}}-y_{m_{1}}, \mathrm{E}_{m_{1}, m_{2}} V_{m_{1}, m_{2}+1}\right\} \\
& =\max \left\{y_{m_{2}}, \mathrm{E}_{m_{1}, m_{2}} V_{m_{1}, m_{2}+1}+y_{m_{1}}\right\}-y_{m_{1}} \\
& =\max \left\{y_{m_{2}}, v^{N-m_{2}, 1}\right\}-y_{m_{1}},
\end{aligned}
$$


where

$$
\begin{array}{rlrl}
v^{N-m_{2}+1,1} & =\mathrm{E}_{m_{1}, m_{2}-1}\left(\max \left\{y_{m_{2}}, v^{N-m_{2}, 1}\right\}\right) \\
& =\mathrm{E}\left(\max \left\{y_{m_{2}}, v^{N-m_{2}, 1}\right\}\right), & & \text { for } m_{1}<m_{2}<N, \\
v^{N-m_{2}+1,1} & =v^{1,1}=\mathrm{E} y_{N}=f_{N}, & & \text { for } m_{2}=N .
\end{array}
$$

Indeed, it follows from (2) that $V_{m_{1}, N}=X_{m_{1}, N}$. Hence, $v^{0,1}=-\infty$.

From (1) we obtain

$$
\begin{aligned}
X_{m_{1}} & =\mathrm{E}_{m_{1}} V_{m_{1}, m_{1}+1} \\
& =\mathrm{E}_{m_{1}}\left(\max \left\{y_{m_{1}+1}, v^{N-m_{1}-1,1}\right\}-y_{m_{1}}\right) \\
& =\mathrm{E}\left(\max \left\{y_{m_{1}+1}, v^{N-m_{1}-1,1}\right\}\right)-y_{m_{1}} \\
& =v^{N-m_{1}, 1}-y_{m_{1}} .
\end{aligned}
$$

In the same way,

$$
\begin{aligned}
V_{m_{1}} & =\max \left\{X_{m_{1}}, \mathrm{E}_{m_{1}} V_{m_{1}+1}\right\} \\
& =\max \left\{v^{N-m_{1}, 1}-y_{m_{1}}, \mathrm{E}_{m_{1}} V_{m_{1}+1}\right\} \\
& =\max \left\{v^{N-m_{1}, 1}-y_{m_{1}}, v^{N-m_{1}, 2}\right\},
\end{aligned}
$$

where

$$
\begin{array}{rlrl}
v^{N-m_{1}+1,2} & =\mathrm{E}_{m_{1}-1}\left(\max \left\{v^{N-m_{1}, 1}-y_{m_{1}}, v^{N-m_{1}, 2}\right\}\right) \\
& =\mathrm{E}\left(\max \left\{v^{N-m_{1}, 1}-y_{m_{1}}, v^{N-m_{1}, 2}\right\}\right), & & \text { for } m_{1}<N-1, \\
v^{N-m_{1}+1,2} & =v^{2,2}=\mathrm{E}\left(\max \left\{f_{N}-y_{N-1}, 0\right\}\right), & & \text { for } m_{1}=N-1 .
\end{array}
$$

Taking into account Theorem 2, we obtain the value $v=v^{N, 2}$.

Now, using Theorem 1, (4), and (5), we obtain the optimal double stopping rule $\tau^{*}=$ $\left(\tau_{1}^{*}, \tau_{2}^{*}\right)$, where

$$
\begin{aligned}
& \tau_{1}^{*}=\min \left\{\min \left\{m_{1}: 1 \leq m_{1} \leq N-1, y_{m_{1}} \leq v^{N-m_{1}, 1}-v^{N-m_{1}, 2}\right\}, N\right\}, \\
& \tau_{2}^{*}=\min \left\{\min \left\{m_{2}: \tau_{1}^{*}<m_{2} \leq N, y_{m_{2}} \geq v^{N-m_{2}, 1}\right\},\left\{N+1: \tau_{1}^{*}=N\right\}\right\} .
\end{aligned}
$$

This completes the proof.

Remark 2. This theorem can easily be generalized when more than two stops are required. In fact, having applied the theorem once to generate two stops, we can treat the time at the second stop as zero and reapply the theorem as many times as necessary.

\section{Examples}

We discuss here three examples in which we specify the distribution of the 'noise' component $\varepsilon$ (i.e. any $\varepsilon_{n}$ ). We assume that the distribution of $\varepsilon$ is either uniform, Laplace (double exponential), or normal; in each case we present a solution of the optimal double stopping problem. 
Example 1. (Uniform distribution.) Let $\varepsilon_{1}, \ldots, \varepsilon_{N}$ be a sequence of independent random variables having uniform distribution $\mathrm{U}(-a, a)$, where $a>0$ is a fixed number. From Theorem 3 we obtain

$$
\begin{aligned}
v^{n, 1} & =\mathrm{E}\left(\max \left\{y_{N-n+1}, v^{n-1,1}\right\}\right) \\
& =\mathrm{E}\left(\max \left\{f_{N-n+1}+\varepsilon_{N-n+1}, v^{n-1,1}\right\}\right) \\
& =\mathrm{E}\left(\max \left\{\varepsilon_{N-n+1}, v^{n-1,1}-f_{N-n+1}\right\}\right)+f_{N-n+1} \\
& =\int_{-a}^{a} \max \left\{x, v^{n-1,1}-f_{N-n+1}\right\}(2 a)^{-1} \mathrm{~d} x+f_{N-n+1} \\
& =\int_{-a}^{c} c(2 a)^{-1} \mathrm{~d} x+\int_{c}^{a} x(2 a)^{-1} \mathrm{~d} x+f_{N-n+1} \\
& =\left(v^{n-1,1}-f_{N-n+1}+a\right)^{2}(4 a)^{-1}+f_{N-n+1},
\end{aligned}
$$

where $c=v^{n-1,1}-f_{N-n+1}, 1 \leq n \leq N-1$, and $v^{0,1}=f_{N}-a$.

Similarly,

$$
\begin{aligned}
v^{n, 2} & =\mathrm{E}\left(\max \left\{v^{n-1,1}-y_{N-n+1}, v^{n-1,2}\right\}\right) \\
& =\mathrm{E}\left(\max \left\{v^{n-1,1}-f_{N-n+1}-\varepsilon_{N-n+1}, v^{n-1,2}\right\}\right) \\
& =\mathrm{E}\left(\max \left\{-\varepsilon_{N-n+1}, v^{n-1,2}-v^{n-1,1}+f_{N-n+1}\right\}\right)+v^{n-1,1}-f_{N-n+1} \\
& =\int_{-a}^{a} \max \{-x, d\}(2 a)^{-1} \mathrm{~d} x+v^{n-1,1}-f_{N-n+1} \\
& =-\int_{-a}^{-d} x(2 a)^{-1} \mathrm{~d} x+\int_{-d}^{a} d(2 a)^{-1} \mathrm{~d} x+v^{n-1,1}-f_{N-n+1} \\
& =\left(v^{n-1,2}-v^{n-1,1}+f_{N-n+1}+a\right)^{2}(4 a)^{-1}+v^{n-1,1}-f_{N-n+1},
\end{aligned}
$$

where $d=v^{n-1,2}-v^{n-1,1}+f_{N-n+1}, 2 \leq n \leq N$, and $v^{1,2}=0$.

In Table 1 we present some numerical results in the case $f_{n}=0.1 n+10, N=6$, and $a=1$. Thus, we get the expected gain $v=v^{6,2}=1.2070$ and $\tau^{*}=\left(\tau_{1}^{*}, \tau_{2}^{*}\right)$, where

$$
\begin{aligned}
& \tau_{1}^{*}=\min \left\{\min \left\{m_{1}: 1 \leq m_{1} \leq 5, y_{m_{1}} \leq v^{6-m_{1}, 1}-v^{6-m_{1}, 2}\right\}, 6\right\}, \\
& \tau_{2}^{*}=\min \left\{\min \left\{m_{2}: \tau_{1}^{*}<m_{2} \leq 6, y_{m_{2}} \geq v^{6-m_{2}, 1}\right\},\left\{7: \tau_{1}^{*}=6\right\} \equiv 7\right\} .
\end{aligned}
$$

For instance, if we observe the sequence

$$
\left\{y_{n}, n=1, \ldots, 6\right\}: 10.0384,9.3296,10.5656,10.6310,10.3470,11.2767,
$$

then $m_{1}=2$ (because $y_{2}=9.3296 \leq v^{4,1}-v^{4,2}=10.9334-0.8484=10.0850$ ) and $m_{2}=6$ (because $y_{6}=11.2767 \geq v^{0,1}=9.6000$ ). This yields the gain $11.2767-9.3296=1.9471$.

TABLE 1: The values for the uniform distribution $\mathrm{U}(-1,1)$.

\begin{tabular}{ccrrrrrr}
\hline$n$ & 0 & \multicolumn{1}{c}{1} & \multicolumn{1}{c}{2} & \multicolumn{1}{c}{3} & \multicolumn{1}{c}{4} & \multicolumn{1}{c}{5} & 6 \\
\hline$v^{n, 1}$ & 9.6000 & 10.6000 & 10.8025 & 10.8918 & 10.9334 & 10.9512 & - \\
$v^{n, 2}$ & - & 0.0000 & 0.3025 & 0.6050 & 0.8484 & 1.0442 & 1.2070 \\
\hline
\end{tabular}


Example 2. (Laplace distribution.) Suppose that independent random variables $\varepsilon_{1}, \ldots, \varepsilon_{N}$ are identically distributed by a Laplace (double exponential) distribution $\mathrm{L}(0, b)$ with probability density function

$$
g(x)=\frac{1}{2 b} \exp \left\{-\frac{|x|}{b}\right\}, \quad x \in(-\infty, \infty), b>0 .
$$

As above, using Theorem 3 we obtain

$$
\begin{aligned}
v^{n, 1} & =\mathrm{E}\left(\max \left\{y_{N-n+1}, v^{n-1,1}\right\}\right) \\
& =\mathrm{E}\left(\max \left\{f_{N-n+1}+\varepsilon_{N-n+1}, v^{n-1,1}\right\}\right) \\
& =\mathrm{E}\left(\max \left\{\varepsilon_{N-n+1}, v^{n-1,1}-f_{N-n+1}\right\}\right)+f_{N-n+1} \\
& =\int_{-\infty}^{\infty} \max \left\{x, v^{n-1,1}-f_{N-n+1}\right\} g(x) \mathrm{d} x+f_{N-n+1} \\
& =\int_{-\infty}^{c} \operatorname{cg}(x) \mathrm{d} x+\int_{c}^{\infty} x g(x) \mathrm{d} x+f_{N-n+1} \\
& =v^{n-1,1}+\frac{b}{2} \exp \left\{\frac{f_{N-n+1}-v^{n-1,1}}{b}\right\},
\end{aligned}
$$

where $c=v^{n-1,1}-f_{N-n+1}, 1 \leq n \leq N-1$, and $v^{0,1}=-\infty$.

Likewise,

$$
\begin{aligned}
v^{n, 2} & =\mathrm{E}\left(\max \left\{v^{n-1,1}-y_{N-n+1}, v^{n-1,2}\right\}\right) \\
& =\mathrm{E}\left(\max \left\{v^{n-1,1}-f_{N-n+1}-\varepsilon_{N-n+1}, v^{n-1,2}\right\}\right) \\
& =\mathrm{E}\left(\max \left\{-\varepsilon_{N-n+1}, v^{n-1,2}-v^{n-1,1}+f_{N-n+1}\right\}\right)+v^{n-1,1}-f_{N-n+1} \\
& =\int_{-\infty}^{\infty} \max \{-x, d\} g(x) \mathrm{d} x+v^{n-1,1}-f_{N-n+1} \\
& =-\int_{-\infty}^{-d} x g(x) \mathrm{d} x+\int_{-d}^{\infty} d g(x) \mathrm{d} x+v^{n-1,1}-f_{N-n+1} \\
& =v^{n-1,2}+\frac{b}{2} \exp \left\{\frac{v^{n-1,1}-v^{n-1,2}-f_{N-n+1}}{b}\right\}
\end{aligned}
$$

where $d=v^{n-1,2}-v^{n-1,1}+f_{N-n+1}, 2 \leq n \leq N$, and $v^{1,2}=0$.

In Table 2, we present some numerical results in the case $f_{n}=100-n, N=5$, and $b=1$. It follows that we have the expected gain $v=v^{5,2}=0.7117$ and $\tau^{*}=\left(\tau_{1}^{*}, \tau_{2}^{*}\right)$, where

$$
\begin{aligned}
& \tau_{1}^{*}=\min \left\{\min \left\{m_{1}: 1 \leq m_{1} \leq 4, y_{m_{1}} \leq v^{5-m_{1}, 1}-v^{5-m_{1}, 2}\right\}, 5\right\}, \\
& \tau_{2}^{*}=\min \left\{\min \left\{m_{2}: \tau_{1}^{*}<m_{2} \leq 5, y_{m_{2}} \geq v^{5-m_{2}, 1}\right\},\left\{6: \tau_{1}^{*}=5\right\} \equiv 6\right\} .
\end{aligned}
$$

TABLE 2: The values for the Laplace distribution $\mathrm{L}(0,1)$.

\begin{tabular}{ccrrrrc}
\hline$n$ & 0 & \multicolumn{1}{c}{1} & \multicolumn{1}{c}{2} & \multicolumn{1}{c}{3} & \multicolumn{1}{c}{4} & 5 \\
\hline$v^{n, 1}$ & $-\infty$ & 95.0000 & 96.3591 & 97.3082 & 98.3069 & - \\
$v^{n, 2}$ & - & 0.0000 & 0.1839 & 0.4031 & 0.5704 & 0.7117 \\
\hline
\end{tabular}


In particular, if we observe the sequence

$$
\left\{y_{n}, n=1, \ldots, 5\right\}: 99.2057,97.1005,96.8307,95.6100,95.7487,
$$

then $m_{1}=5$ and $m_{2}=6$. We buy nothing, so the gain is $v=0$.

Example 3. (Normal distribution.) Let $\varepsilon_{1}, \ldots, \varepsilon_{N}$ be a sequence of independent random variables having normal distribution $\mathrm{N}\left(0, \sigma^{2}\right)$ with probability density function

$$
h(x)=\frac{1}{\sqrt{2 \pi} \sigma} \exp \left\{-\frac{x^{2}}{2 \sigma^{2}}\right\}, \quad x \in(-\infty, \infty), \sigma>0 .
$$

As before, from Theorem 3 we obtain

$$
\begin{aligned}
v^{n, 1} & =\mathrm{E}\left(\max \left\{y_{N-n+1}, v^{n-1,1}\right\}\right) \\
& =\mathrm{E}\left(\max \left\{f_{N-n+1}+\varepsilon_{N-n+1}, v^{n-1,1}\right\}\right) \\
& =\mathrm{E}\left(\max \left\{\varepsilon_{N-n+1}, v^{n-1,1}-f_{N-n+1}\right\}\right)+f_{N-n+1} \\
& =\int_{-\infty}^{\infty} \max \left\{x, v^{n-1,1}-f_{N-n+1}\right\} h(x) \mathrm{d} x+f_{N-n+1} \\
& =\int_{-\infty}^{c} \operatorname{ch}(x) \mathrm{d} x+\int_{c}^{\infty} x h(x) \mathrm{d} x+f_{N-n+1} \\
& =c \Phi\left(\frac{c}{\sigma}\right)+\sigma \varphi\left(\frac{c}{\sigma}\right)+f_{N-n+1} \\
& =\sigma \psi\left(\frac{v^{n-1,1}-f_{N-n+1}}{\sigma}\right)+f_{N-n+1},
\end{aligned}
$$

where $\psi(x)=\varphi(x)+x \Phi(x), \varphi(x)$ is the density function of the standard normal distribution, $\Phi(x)$ is the distribution function of the standard normal distribution, $c=v^{n-1,1}-f_{N-n+1}$, $1 \leq n \leq N-1$, and $v^{0,1}=-\infty$.

In the same way,

$$
\begin{aligned}
v^{n, 2} & =\mathrm{E}\left(\max \left\{v^{n-1,1}-y_{N-n+1}, v^{n-1,2}\right\}\right) \\
& =\mathrm{E}\left(\max \left\{v^{n-1,1}-f_{N-n+1}-\varepsilon_{N-n+1}, v^{n-1,2}\right\}\right) \\
& =\mathrm{E}\left(\max \left\{-\varepsilon_{N-n+1}, v^{n-1,2}-v^{n-1,1}+f_{N-n+1}\right\}\right)+v^{n-1,1}-f_{N-n+1} \\
& =\int_{-\infty}^{\infty} \max \{-x, d\} h(x) \mathrm{d} x+v^{n-1,1}-f_{N-n+1} \\
& =-\int_{-\infty}^{-d} x h(x) \mathrm{d} x+\int_{-d}^{\infty} d h(x) \mathrm{d} x+v^{n-1,1}-f_{N-n+1} \\
& =\sigma \varphi\left(\frac{d}{\sigma}\right)+d \Phi\left(\frac{d}{\sigma}\right)+v^{n-1,1}-f_{N-n+1} \\
& =\sigma \psi\left(\frac{v^{n-1,2}-v^{n-1,1}+f_{N-n+1}}{\sigma}\right)+v^{n-1,1}-f_{N-n+1},
\end{aligned}
$$

where $d=v^{n-1,2}-v^{n-1,1}+f_{N-n+1}, 2 \leq n \leq N$, and $v^{1,2}=0$. 
TABLE 3: The values for the normal distribution $\mathrm{N}(0,1)$.

\begin{tabular}{ccccccccc}
\hline$n$ & 0 & 1 & 2 & 3 & 4 & 5 & 6 & 7 \\
\hline$v^{n, 1}$ & $-\infty$ & 3.0615 & 5.6583 & 7.4079 & 8.1709 & 8.2954 & 8.2967 & - \\
$v^{n, 2}$ & - & 0.0000 & 0.0015 & 0.0183 & 0.1841 & 0.9497 & 2.6573 & 5.2368 \\
\hline
\end{tabular}

In Table 3, we present some numerical results in the case $f_{n}=8 \sin (\pi n / 8), N=7$, and $\sigma=1$. It follows that the expected gain is $v=v^{7,2}=5.2368$ and $\tau^{*}=\left(\tau_{1}^{*}, \tau_{2}^{*}\right)$, where

$$
\begin{aligned}
& \tau_{1}^{*}=\min \left\{\min \left\{m_{1}: 1 \leq m_{1} \leq 6, y_{m_{1}} \leq v^{7-m_{1}, 1}-v^{7-m_{1}, 2}\right\}, 7\right\}, \\
& \tau_{2}^{*}=\min \left\{\min \left\{m_{2}: \tau_{1}^{*}<m_{2} \leq 7, y_{m_{2}} \geq v^{7-m_{2}, 1}\right\},\left\{8: \tau_{1}^{*}=7\right\} \equiv 8\right\} .
\end{aligned}
$$

For instance, if we observe the sequence

$$
\left\{y_{n}, n=1, \ldots, 7\right\}: 2.4894,5.9438,7.0311,8.9202,7.8443,5.4808,3.5506,
$$

then $m_{1}=1$ (because $y_{1}=2.4894 \leq v^{6,1}-v^{6,2}=8.2967-2.6573=5.6394$ ) and $m_{2}=4$ (because $y_{4}=8.9202 \geq v^{3,1}=7.4079$ ). This yields the gain $8.9202-2.4894=6.4308$.

\section{Acknowledgements}

The authors are grateful to Professor M. L. Nikolaev for a useful discussion on the results of this paper, and to the referee for helpful remarks. The authors acknowledge the support of the Australian Research Council (grant number DP0556631). The research of the first author was supported by the Ministry of Education and Science of the Russian Federation through the programme 'Development of Scientific Potential of Higher Education'.

\section{References}

[1] Chow, Y. S., Robins, H. And Siegmund, D. (1971). Great Expectations: The Theory of Optimal Stopping. Houghton Mifflin, Boston, MA.

[2] Haggstrom, G. W. (1966). Optimal stopping and experimental design. Ann. Math. Statist. 37, 7-29.

[3] Nikolaev, M. L. (1999). On optimal multiple stopping of Markov sequences. Theory Prob. Appl. 43, $298-306$.

[4] Shiryaev, A. N. (1999). Essentials of Stochastic Finance: Facts, Models, Theory. World Scientific, Singapore. 